\title{
Domain 36 of Tropoelastin in Elastic Fiber Formation
}

\author{
Risa Nonaka, ${ }^{a, b, \#}$ Fumiaki Sato, ${ }^{a, \#}$ and Hiroshi Wachi*,a \\ ${ }^{a}$ Department of Clinical Chemistry, Hoshi University School of Pharmacy and Pharmaceutical Sciences; 2-4-41 \\ Ebara, Shinagawa-ku, Tokyo 142-8501, Japan: and ${ }^{b}$ Research Institute for Diseases of Old Age, Juntendo University \\ Graduate School of Medicine; 2-1-1 Hongo, Bunkyo-ku, Tokyo 113-8421, Japan. \\ Received December 2, 2013; accepted January 17, 2014
}

Elastic fiber assembly is a complex stepwise process involving multiple different proteins and enzymes. Domain 36, encoded by the last exon of the elastin gene, is recognized to be an important domain for deposition onto microfibrils, an essential step in elastic fiber assembly. However, the role of domain 36 in elastic fiber assembly has not been clarified. Here, we utilized our established in vitro assembly model to identify the importance of domain 36 for the assembly process. Our results showed that the lack of domain 36 in bovine tropoelastin results in deficient elastic fiber assembly. A similar result was obtained with the point mutation of two cysteine residues and the deletion of the Lysine-Arginine-Lysine-Arginine (RKRK) sequence in domain 36. Double immunofluorescence of tropoelastin and fibrillin-1, a main component of microfibrils, demonstrated reduced localization of these mutant tropoelastin molecules on fibrillin-1 fibers. Moreover, the binding affinity of these mutants to fibrillin-1 and microfibril-associated glycoprotein (MAGP) was significantly decreased. These data indicate that domain 36 of tropoelastin facilitates elastic fiber assembly by interacting with microfibrils via two cysteine residues and the RKRK sequence.

Key words elastic fiber formation; tropoelastin; microfibril

Elastin is a highly insoluble extracellular matrix protein and the core protein constituting elastic fibers. Elastin is secreted from cells as a soluble protein referred to as tropoelastin. The assembly of tropoelastin into a fibrillar matrix is a complex stepwise process. In the first step, secreted tropoelastin molecules self-aggregate via coacervation. ${ }^{1)}$ The tropoelastin aggregates are then deposited onto preformed microfibrillar templates, which act as a molecular scaffold. ${ }^{2)}$ Finally, the oxidative deamination of peptidyl lysine residues in tropoelastin is catalyzed by lysyl oxidase. The cross-linking of tropoelastin forms elastin, which contributes to the elasticity of tissues, such as the skin, aorta and lungs, as a component of mature elastic fibers. However, the precise mechanisms underlying elastic fiber assembly remain unknown.

Functional mapping studies, including our previous studies, have identified domains in the C-terminal half of tropoelastin (domain 16-36) that facilitate fiber assembly. Domain 16 and 30 of the hydrophobic domain play important roles in the formation of the $\beta$-sheet structure, ${ }^{3-5)}$ which is critical for fiber formation and the self-assembly of tropoelastin. ${ }^{6)}$ Further, the study using surface plasmon resonance showed that the $\mathrm{N}$ terminal region of tropoelastin, encompassing domains 2-18 binds to the $\mathrm{N}$-terminus of fibrillin-1, a main component of microfibrils. ${ }^{7)}$

Domain 36 encoded by exon 36, the last exon in elastin gene, is highly conserved ${ }^{8)}$ and interacts with integrins ${ }^{9-11)}$ and sulfated proteoglycans. ${ }^{12)}$ Peptide binding analyses have implicated the Lysine-Lysine-Arginine-Lysine-Arginine (GRKRK) sequence and the last 17 amino acids at the C-terminus of bovine tropoelastin to play a role in the human skin fibroblast and bovine chondrocyte adhesive activity. ${ }^{10,12)}$ Furthermore, domain 36 has been recognized to be an important domain for the formation of elastic fiber, as antibodies directed to domain 36 prevent elastin assembly, ${ }^{13)}$ and deleting

The authors declare no conflict of interest.

\# These authors contributed equally to this work. this sequence results in a dramatic reduction in the levels of cross-linked elastin in in vitro culture systems. ${ }^{14)}$ However, there is no direct evidence as to how domain 36 of tropoelastin is involved in elastic fiber assembly.

In the present study, we demonstrated the importance of domain 36 of tropoelastin for elastic fiber assembly using our established in vitro model, ${ }^{15}$ ) which enables the quantitative comparison of various tropoelastin molecules.

\section{MATERIALS AND METHODS}

Purification of Recombinant Tropoelastin Molecules Recombinant bovine tropoelastin was prepared as previously described. ${ }^{15)}$ In order to generate the C-terminal mutants, bovine elastin gene templates were amplified using the primers listed in Table 1 (BTEex2S was commonly used, all mutant, anti-sense primers were used: C750/755-AS for C750/755 A, RK-AS for $\triangle$ RKRK and Ex35AS for $\Delta 36$ ). These products were inserted into a bacterial expression pTrcHis-TOPO vector (Invitrogen). The integrity of the constructs was confirmed using a DNA sequence analysis. Each recombinant tropoelastin molecule was obtained via overexpression from the plasmid and purified according to the same method as that used for bovine tropoelastin (BTE). The purified recombinant proteins were resuspended in sodium dodecyl sulfate-polyacrylamide gel electrophoresis (SDS-PAGE) sample buffer includ-

Table 1. Primers List Using in This Study

\begin{tabular}{lc}
\hline \hline Primer & Primer sequence \\
\hline BTEex2S & 5'-GGAGGGGTCCCAGGAGCT-3' \\
C750/755 A-AS & 5'-TCACTTTCTCTTCCGGCCGGC- \\
& GGATTTCCCCAGGGCGGCCCCACC-3' \\
RK-AS & 5'-TCAGCCACAGGATTTCCCCAG-3' \\
Ex35AS & 5'-TCATGGGAATCCCTGGGC-3' \\
MAGP-S & 5'-ATGAGAGCTGCCTACCTCTT-3' \\
MAGP-AS & 5'-GCAGCTCCCACAGCTCCTG-3' \\
\hline
\end{tabular}


ing $100 \mathrm{~mm}$ of dithiothreitol. Samples were run on SDS-PAGE gels and subjected to a Western blot analysis using anti-bovine tropoelastin antibody (BA4; Sigma).

Treatment with Recombinant Tropoelastin Molecules ARPE-19 cells (ATCC) derived from human retinal pigment epithelium were grown in Dulbecco's modified eagle medium supplemented with L-glutamine and penicillin/streptomycin (Invitrogen) as well as $10 \%(\mathrm{v} / \mathrm{v})$ cosmic calf serum (Hyclone Laboratories). ARPE-19 cells were plated on eight-well
LabTek chamber slides (Nunc) or 60-mm dishes (BD) for immunofluorescence or the determination of cross-linked amino acids, respectively. After two days in confluent culture, the cells were treated with the recombinant tropoelastin molecules for two to eight days. The medium was changed every two days.

Immunofluorescence and Determination of CrossLinked Amino Acids in the Matrix The methods used for the immunofluorescence study and determination of des-

A

B

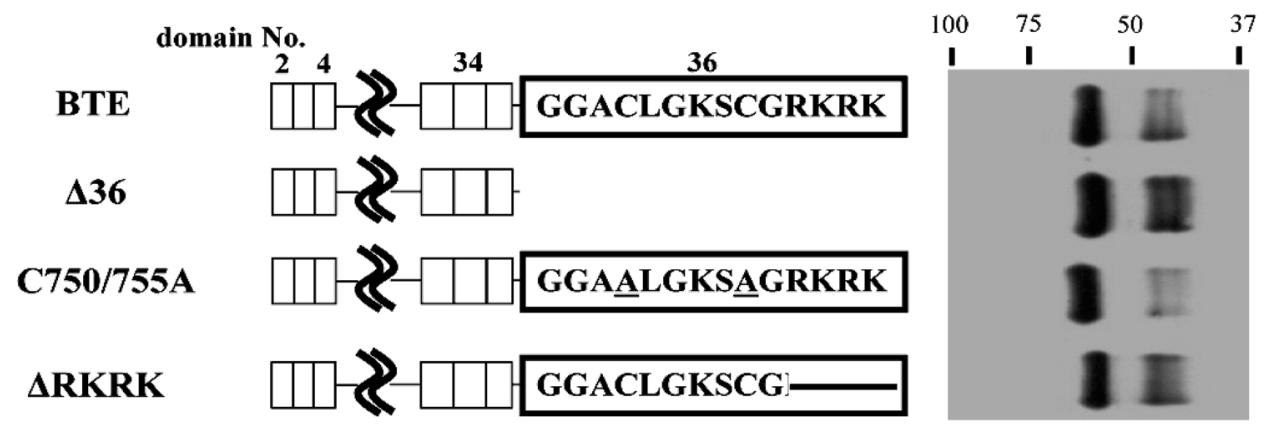

Fig. 1. Recombinant Tropoelastin Construction Used in This Study

(A) Scheme of tropoelastin constructs used in this study. (B) Purified recombinant tropoelastins were detected by Western blotting with BA4 antibody.

A

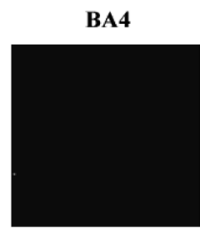

FBN-1

ARPE-19

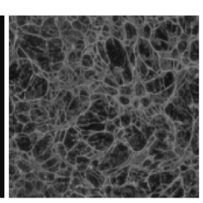

2d

4d

6d

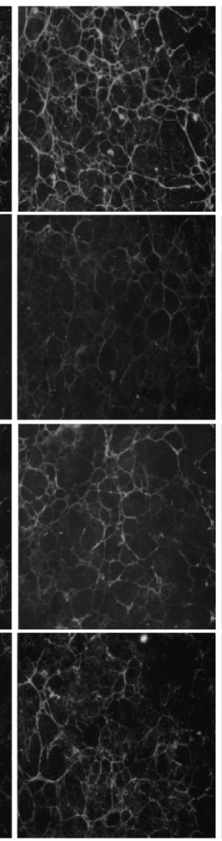

8d

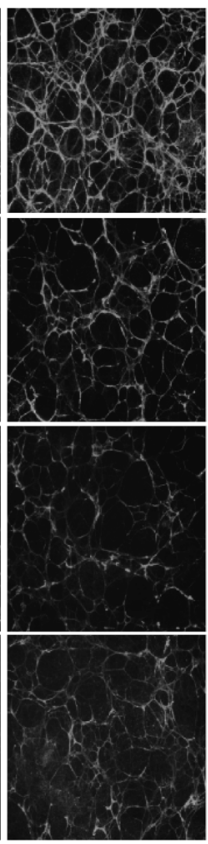

B

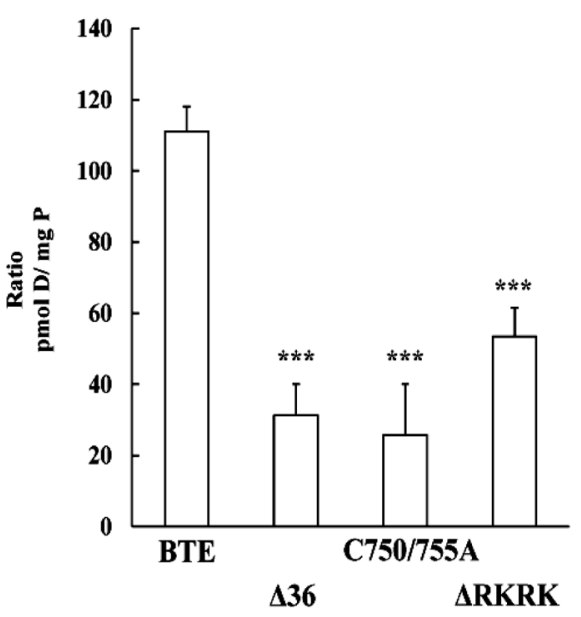

Fig. 2 Elastic Fiber Assembly with Recombinant Tropoelastins

(A) Double immunofluorescence of 8-d cultured ARPE-19 cells with BA4 and FBN-1 antibodies (upper panels; ARPE-19). After 2d in confluent culture, ARPE-19 cells were treated with recombinant tropoelastins at concentration of $1.6 \times 10^{-7} \mathrm{M}$ for $2-8 \mathrm{~d}$. At the end of the incubation period, the cells were immunostained with BA4 antibody (bottom panels). Magnification; $\times 200$. (B) ARPE-19 cells were treated with recombinant tropoelastins for $8 \mathrm{~d}$ at concentration of $1.6 \times 10^{-7} \mathrm{M}$. Desmosine (pmol D) was detected by radioimmunoassay and normalized to total protein (mg P). $n=6,{ }^{* * *} p<0.001$ vs. BTE 
mosine content have been described in a previous report. ${ }^{15)}$ For immunofluorescence, at the end of the treatment with recombinant tropoelastin, the cells were fixed and incubated with anti-BA4 and/or anti-fibrillin-1 (FBN-1; Elastin Products Company) antibodies for one hour, followed by several washes with phosphate buffered saline (PBS) and a second round of incubation with anti-mouse or rabbit immunoglobulin $\mathrm{G}(\mathrm{IgG})$ conjugated Alexa Fluor 488 or 546 (Invitrogen). In order to determine the desmosine content, ARPE-19 cells were treated with recombinant tropoelastin for eight days. The desmosine levels in the hydrolysates were determined using a radioimmunoassay. ${ }^{16)}$ The desmosine levels (pmol) were normalized to those of the total protein $(\mathrm{mg})$ in the dishes.
Solid Phase Binding Assay Microfibril-associated glycoprotein (MAGP) was constructed via reverse transcription polymerase chain reaction (RT-PCR) amplification of total RNA isolated from human aortic smooth muscle cells using the following primers listed in Table 1. Previously reported PET cDNA was kindly provided by Dr. Robert P. Mecham (Washington University). MAGP and PET cDNA was inserted into the pcDNA3.1/V5-His-TOPO expression vector (Invitrogen), which fuses a V5 and His tags to the C-terminal upon insert. Recombinant PET-V5 and MAGP-V5 were prepared using the same methods as described previously. ${ }^{4}$ A solid phase binding assay was also performed as described. ${ }^{17)}$ Micro-titer plates were coated at $4^{\circ} \mathrm{C}$ overnight with equal

A

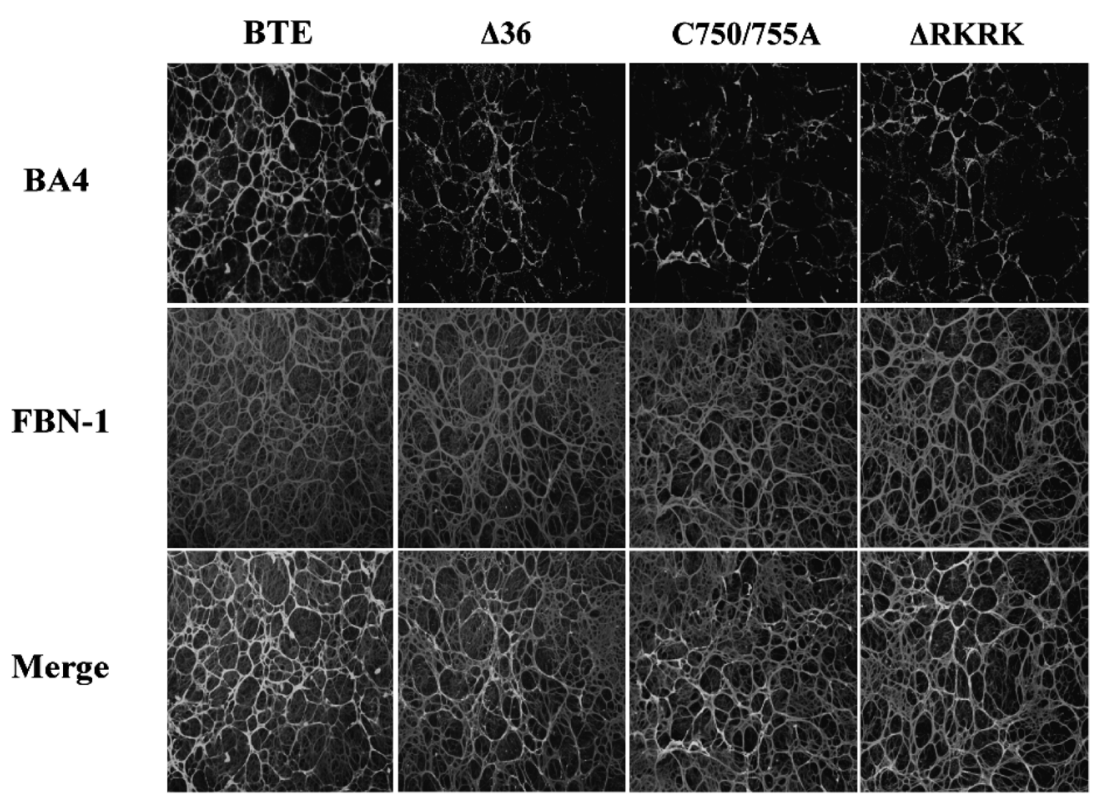

B

Fibrillin-1

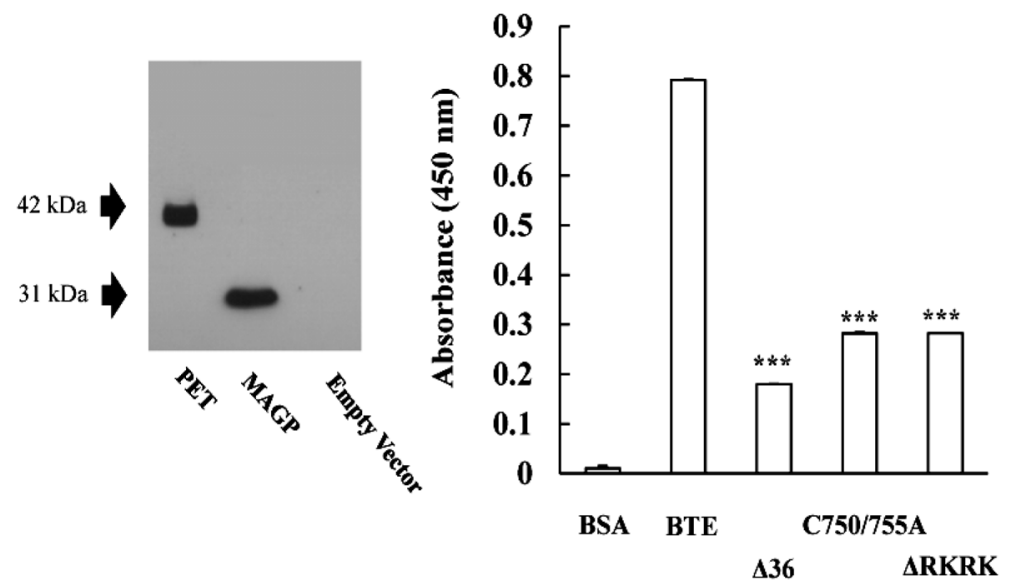

MAGP

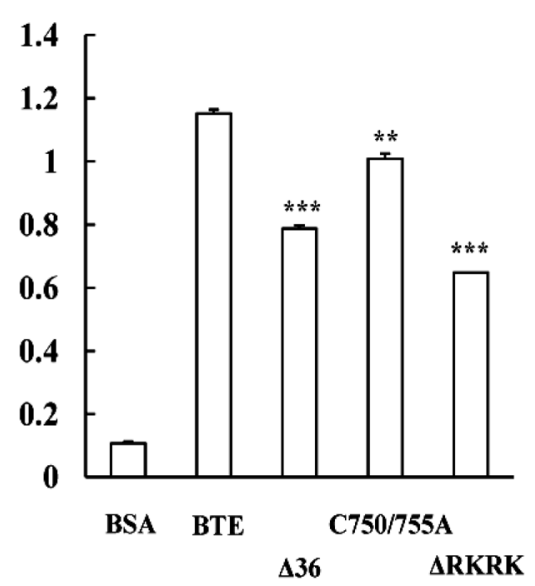

Fig. 3. Deposition of Tropoelastin onto Microfibrils and Interaction with Microfibrillar Components

(A) Double immunofluorescence of ARPE-19 cells with BA4 and $\mathrm{FBN}-1$ antibodies after treatment with recombinant tropoelastins at concentration of $3.2 \times 10^{-7} \mathrm{M}$ for $8 \mathrm{~d}$. (B) Purified recombinant PET-V5 or MAGP-V5, which have C-terminally fused V5 and His tags, were detected by Western blot assay using anti-V5 antibody (left panel). The biding assay for each tropoelastin with PET-V5 or MAGP-V5 was performed by solid phase binding assay using anti-V5 antibody (right graphs). $n=6$, ${ }^{* * *} p<0.001$ vs. BTE. ${ }^{* *} p<0.01$ vs. BTE. 
amounts $\left(1.6 \times 10^{-7} \mathrm{M}\right)$ of each recombinant tropoelastin molecule or bovine serum albumin (BSA) in bicarbonate buffer, respectively. Tropoelastin bound-PET-V5 and -MAGP-V5 were detected using anti-V5-epitope antibodies. Bound protein was quantified using a colorimetric assay using the TMB-substrate reagent. The plates were read at a wavelength of $450 \mathrm{~nm}$.

Statistical Analysis Data were statistically analyzed using unpaired $t$-tests (Microsoft Exel XP). The results were considered statistically significant when the $p$ value was $<0.05$. All data are shown as the mean value \pm S.E.M. (standard error of the mean).

\section{RESULTS AND DISCUSSION}

Domain 36 contains the molecule's only 2 Cys residues and a terminal Arg-Lys-Arg-Lys (RKRK) sequence. The cysteine residues have been shown to form a disulfide-bonded loop structure that creates a highly charged "pocket" at the end of the molecule believed to facilitate interactions between tropoelastin and highly acidic microfibrils. ${ }^{18)}$ To clarify the importance of this region of the protein to elastin assembly, we generated C-terminal mutants of full-length BTE in which domain 36 was deleted in its entirety $(\Delta 36)$ and both of the cysteines residues were mutated to alanines (C750/755 A), as well as one construct in which the RKRK sequence was deleted ( $\triangle$ RKRK) (Fig. 1A). Immunoblotting with BA4 antibody showed that purified recombinant tropoelastins gave major bands of about $60 \mathrm{kDa}$ as predicted from amino acid sequence analysis (BTE and C750/755 A; 61 kDa, $\Delta 36$ and $\Delta \mathrm{RKRK} ; 60 \mathrm{kDa}$ ) with the degraded products (Fig. 1B). Next, we assessed the ability of these mutants to precipitate in elastic fiber assembly using our in vitro model. ARPE-19 cells were reported previously to express microfibrillar proteins but not tropoelastin. ${ }^{15)}$ This was confirmed by immunostaining of ARPE-19 cells with BA4 and FBN-1 antibodies (Fig. 2A upper panels; ARPE-19). When each recombinant tropoelastin molecule was treated with ARPE-19 cells for 2-8d, immunofluorescence labeling using BA4 antibody showed that these mutants formed mesh-like structure in a time-dependent manner (Fig. 2A). However, the assembly of C-terminal mutants significantly decreased compared to BTE. Quantitative assembly assay also showed that the amount of desmosine, specific marker of elastic fiber, in ARPE-19 cells treated with C-terminal mutants for $8 \mathrm{~d}$, significantly decreased (Fig. 2B). These results suggest that domain 36 including two cysteine residues and RKRK amino sequence in domain 36 is an important domain/sequence for elastic fiber assembly.

Microfibrillar components, such as fibrillin-1 and MAGP, are probably served as scaffold to direct tropoelastin crosslinking and consequential elastic fiber formation. To confirm the deposition of tropoelastin mutants onto microfibril fiber, we performed double immunofluorescence of BA4 and FBN-1 antibodies in ARPE-19 cells treated with each recombinant tropoelastin. The formation of fibrillin-1 fibers in the cells treated with each tropoelastin remains unchanged. The colocalizaiton of mutant recombinants with microfibril fibers was dramatically decreased compared with BTE (Fig. 3A), suggesting that two cysteine residues and RKRK amino sequence in domain 36 involves tropoelastin deposition onto microfibrils. Therefore, to determine the molecular interactions between $\mathrm{C}$-teminal mutants and microfibrils, we performed a solid phase binding assay using recombinant PET-V5, known to binding region of fibrillin-1 to tropoelastin, and MAGP-V5. PET-V5 and MAGP-V5 from condition medium were detected as proteins of approximately 42 and $31 \mathrm{kDa}$ by Western blot assay using anti-V5 antibody, respectively (Fig. 3B left panel). The binding assay using anti-V5 antibody showed lower binding affinity of C-terminal mutants to PET-V5 and MAGP-V5 (Fig. 3B). Especially, the binding of C-terminal mutants to PET dramatically decreased. These results indicated that domain 36 involved the interaction with microfibrils via two cysteine residues and RKRK sequence, at least fibrillin-1 and MAGP.

In conclusion, we clarified domain 36 of tropoelastin contributes to the binding to fibrillin-1 and MAGP through two cysteine residues and RKRK sequence, resulting in the promotion of elastic fiber assembly.

Acknowledgments We thank Dr. Barry C. Starcher (University of Texas Health Center at Tyler) for measurement of desmosine. This study is supported by MEXT-Supported Program for the Strategic Research Foundation at Private Universities, 2008-2012 and the Grant-in-Aid for Young Scientists (B) (F. Sato and R. Nonaka).

\section{REFERENCES}

1) Vrhovski B, Jensen S, Weiss AS. Coacervation characteristics of recombinant human tropoelastin. Eur. J. Biochem., 250, 92-98 (1997).

2) Ross R, Fialkow RJ, Altman LK. The morphogenesis of elastic fibers. Adv. Exp. Med. Biol., 79, 7-17 (1977).

3) Sato F, Wachi H, Ishida M, Nonaka R, Onoue S, Urban Z, Starcher $\mathrm{BC}$, Seyama Y. Distinct steps of cross-linking, self-association, and maturation of tropoelastin are necessary for elastic fiber formation. J. Mol. Biol., 369, 841-851 (2007).

4) Wachi H, Sato F, Nakazawa J, Nonaka R, Szabo Z, Urban Z, Yasunaga T, Maeda I, Okamoto K, Starcher BC, Li DY, Mecham RP, Seyama Y. Domains 16 and 17 of tropoelastin in elastic fibre formation. Biochem. J., 402, 63-70 (2007).

5) Kozel BA, Wachi H, Davis EC, Mecham RP. Domains in tropoelastin that mediate elastin deposition in vitro and in vivo. J. Biol. Chem., 278, 18491-18498 (2003).

6) Robson P, Wright GM, Sitarz E, Maiti A, Rawat M, Youson JH, Keeley FW. Characterization of lamprin, an unusual matrix protein from lamprey cartilage. Implications for evolution, structure, and assembly of elastin and other fibrillar proteins. J. Biol. Chem., 268, 1440-1447 (1993).

7) Clarke AW, Wise SG, Cain SA, Kielty CM, Weiss AS. Coacervation is promoted by molecular interactions between the PF2 segment of fibrillin-1 and the domain 4 region of tropoelastin. Biochemistry, 44, 10271-10281 (2005).

8) Chung MI, Miao M, Stahl RJ, Chan E, Parkinson J, Keeley FW. Sequences and domain structures of mammalian, avian, amphibian and teleost tropoelastins: Clues to the evolutionary history of elastins. Matrix Biology: Journal of the International Society for Matrix Biology, 25, 492-504 (2006).

9) Rodgers UR, Weiss AS. Integrin alpha v beta 3 binds a unique nonRGD site near the C-terminus of human tropoelastin. Biochimie, $\mathbf{8 6}$, 173-178 (2004).

10) Bax DV, Rodgers UR, Bilek MM, Weiss AS. Cell adhesion to tropoelastin is mediated via the C-terminal GRKRK motif and integrin alphaVbeta3. J. Biol. Chem., 284, 28616-28623 (2009).

11) Lee P, Bax DV, Bilek MM, Weiss AS. A novel cell adhesion region in tropoelastin that mediates attachment to integrin alphaVbeta5. J. Biol. Chem., 289, 1467-1477 (2014). 
12) Broekelmann TJ, Kozel BA, Ishibashi H, Werneck CC, Keeley FW, Zhang L, Mecham RP. Tropoelastin interacts with cell-surface glycosaminoglycans via its COOH-terminal domain. J. Biol. Chem., 280, 40939-40947 (2005).

13) Brown-Augsburger P, Broekelmann T, Rosenbloom J, Mecham RP. Functional domains on elastin and microfibril-associated glycoprotein involved in elastic fibre assembly. Biochem. J., 318, 149-155 (1996).

14) Hsiao H, Stone PJ, Toselli P, Rosenbloom J, Franzblau C, Schreiber BM. The role of the carboxy terminus of tropoelastin in its assembly into the elastic fiber. Connect. Tissue Res., 40, 83-95 (1999).

15) Wachi H, Sato F, Murata H, Nakazawa J, Starcher BC, Seyama Y. Development of a new in vitro model of elastic fiber assembly in human pigmented epithelial cells. Clin. Biochem., 38, 643-653
(2005).

16) Starcher BC, Mecham RP. Desmosine radioimmunoassay as a means of studying elastogenesis in cell culture. Connect. Tissue Res., 8, 255-258 (1981).

17) Trask TM, Trask BC, Ritty TM, Abrams WR, Rosenbloom J, Mecham RP. Interaction of tropoelastin with the amino-terminal domains of fibrillin-1 and fibrillin-2 suggests a role for the fibrillins in elastic fiber assembly. J. Biol. Chem., 275, 24400-24406 (2000).

18) Brown PL, Mecham L, Tisdale C, Mecham RP. The cysteine residues in the carboxy terminal domain of tropoelastin form an intrachain disulfide bond that stabilizes a loop structure and positively charged pocket. Biochem. Biophys. Res. Commun., 186, 549-555 (1992). 